\title{
Duosensor zur simultanen Erfassung der akralen Wiederenwärmung und Wiederdurchblutung
}

\author{
M. Scheiner, F. Schuster und O. Hochmuth \\ Institut für Elektrotechnik der Humboldt-Universität zu Berlin
}

\begin{abstract}
Einleitung
In der klinischen Diagnostik findet die Messung der akralen Wiedererwärmung der Haut zunehmend Anwendung, unter anderem bei der Diagnose von Neurodermitis, Sklerodermie und endogenen Ekzemen [4]. Bei diesem Meßverfahren werden Teile der Extremitäten einer kontrollierten Unterkühlung ausgesetzt. Im Anschluß werden Temperatur und Durchblutung der Haut während der Wiedererwärmung des Gewebes registriert. Aus dem zeitlichen Verlauf dieser Daten können Erkenntnisse über die Beschaffenheit der Haut und deren Durchblutung gewonnen werden. Die geringe Belastung des Patienten durch dieses nichtinvasive Verfahren erlaubt es, häufige Messungen durchzuführen, beispielsweise um den Verlauf einer Therapie überwachen zu können.
\end{abstract}

\section{Methoden}

Der hier beschriebene Duosensor ermöglicht die gleichzeitige Erfassung von Temperatur und Durchblutungszustand der Haut bei der akralen Wiedererwärmungsmessung. Das in Abb. 1 dargestellte Gerät ist für Messungen am Finger (vorzugsweise am Zeigefinger) ausgelegt. Ein neuartiges Sensorkonzept ermöglicht die berührungslose Erfassung von Temperatur und Durchblutungszustand.

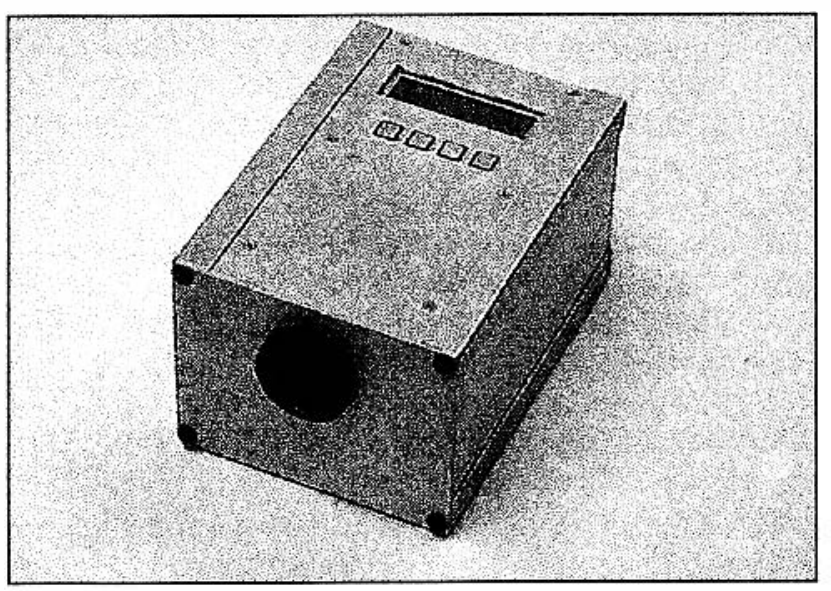

Abbildung 1: $\quad$ Ansicht des Duosensors

Das Gerät beinhaltet sowohl die Sensoren zur Gewinnung der Meßwerte als auch die notwendige Elektronik zur Auswertung der Daten. Der eingebaute Mikrocontroller (SAB 80C537) gestattet in Verbindung mit der LCD-Anzeige die Darstellung der Ergebnisse direkt im Anschluß an die Messung. Die Bedienung des Duosensors wird durch eine übersichtliche Menütechnik vereinfacht. Bei Bedarf können sämtliche Daten über die eingebaute serielle Schnittstelle zu einem PC, Plotter oder Drucker übertragen werden. Alle vom Anwender vorgenommenen Einstellungen sowie das Ergebnis der letzten Messung bleiben auch nach dem Abschalten der Betriebsspannung erhalten und stehen beim nächsten Einsatz wieder zur Verfügung. Umfangreiche Funktionen zum Systemtest helfen, Fehler in den Baugruppen des Duosensors schnell zu lokalisieren.

Die berührungslose Bestimmung der Hauttemperatur durch die Erfassung der emittierten Infrarotstrahlung hat bereits mehrfach ihre Eignung für die klinische Praxis gezeigt [1, 4]. Für den Duosensor wurde nach umfangreichen Untersuchungen ein Sensor der Firma Exergen ausgewählt. Er zeichnet sich durch die geringe Baugröße, einen optimalen Meßfleckdurchmesser und gute Übereinstimmung mit dem erforderlichen Temperatur- und Spektralbereich aus. Das Ausgangssignal des Infrarot-Thermoelementes wird in einem integrierten Thermoelementverstärker (AD 595) aufbereitet und über einen Antialiasingtiefpaß dem Mikrocontroller zugeführt. Dieser realisiert die Abtastung (Abtastfrequenz $1 \mathrm{~Hz}$ ), die Analog-Digital-Umsetzung und die weitere Signalverarbeitung. Die Temperaturmessung erfolgt am mittleren Fingerglied des Zeigefingers.

Für die berührungslose Ermittlung des Durchblutungszustándes wurde ein Sensor entwickelt, der auf dem Prinzip der Photoplethysmographie beruht. Als Lichtquelle dient eine Infrarotemitterdiode vom Typ VX 301 mit einer maximalen Emission bei $895 \mathrm{~nm}$. Das durch die Fingerkuppe transmittierte Infrarotlicht gelangt auf einen Sensor, der zur Erhöhung der Empfindlichkeit aus vier parallelgeschalteten Photodioden (BPW 34) besteht. Der beleuchtungsabhängige Photostrom wird in einen Spannungswert gewandelt. Dieser besteht aus einem Gleich- und einem Wechselanteil [3]. Der Gleichanteil steht nach der Digitalisierung für eine Auswertung zur Verfügung. Als Folge der pulssynchronen Ǎnderung des Blutvolumens im Finger entsteht der Wechselanteil. Er wird verstärkt und dem Antialiasingtiefpaß zugeführt. Der verwendete integrierte Tiefpaß (MAX 291) stellt ein Filter 8. Ordnung mit Butterworth-Charakteristik dar. Die Grenzfrequenz beträgt $40 \mathrm{~Hz}$ (nach [2]). Im Mikrocontroller erfolgt die Analog-Digital-Umsetzung des Signals mit einer Abtastrate von $200 \mathrm{~Hz}$.

Eine vom Mikrocontroller angesteuerte Konstantstromquelle erzeugt den Strom, der durch die 
Infrarotemitterdiode fließt. Zu Beginn der Durchblutungsmessung erfolgt in einer Anpassungsphase die automatische Adaption der Lichtintensität an die optischen Eigenschatten des jeweiligen Zeigefingers. Damit kann eine Übersteuerung bzw. ein geringer Signal-Rausch-Abstand vermieden werden.

Die Baugruppe zur Aufnahme der Sensoren bildet zusammen mit den drei Leiterplatten (Digitalschaltung. Analogschaltung, Tastatur und Anzeige) und der Deckplatte eine konstruktive Einheit, welche in Abb. 2 dargestellt ist.

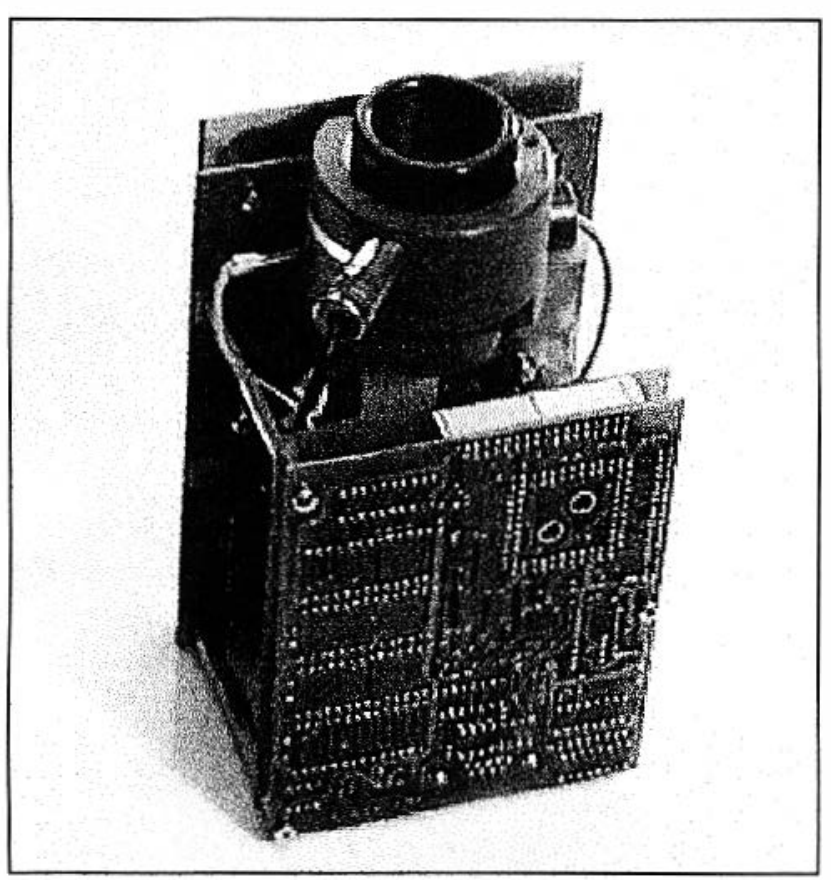

Abbildung 2: $\quad$ Aufbau des Duosensors

\section{Ergebnisse}

Abb. 3 zeigt einen mit dem Duosensor registrierten Verlauf der Wiedererwärmung. Der Vorgang kann

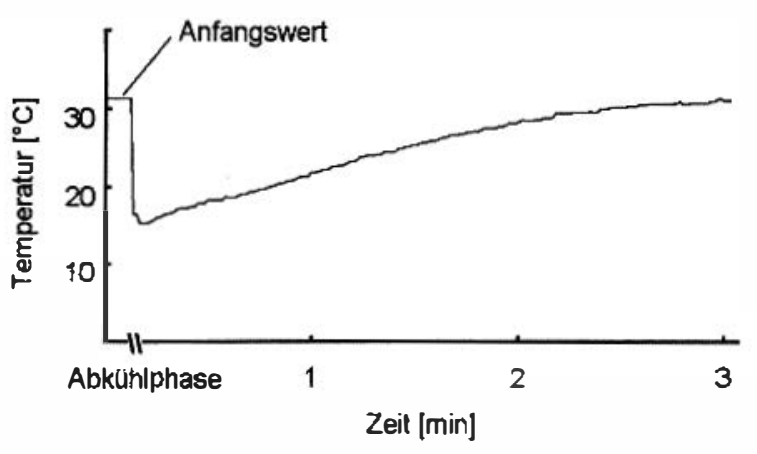

Abbildung 3: $\quad$ Typischer Verlauf der Wiedererwärmung

durch die Sprungantwort eines Verzögerungsgliedes 1. Ordnung beschrieben werden [4]. Zu dessen Charakterisierung wird der Kennwert $\tau$ vom Duosensor ermittelt.
Ein Beispiel für den Verlauf der Durchblutung nach Kälteschock ist in Abb. 4 dargestellt. Es ist zu erkennen, daß die auftretenden Durchblutungswerte über dem Anfangsniveau liegen können.

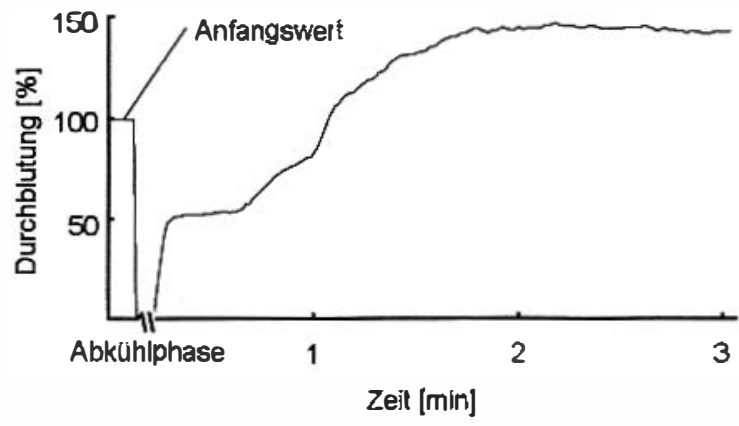

Abbildung 4: Typischer Verlauf der Wiederdurchblutung

\section{Diskussion}

Mit dem Duosensor steht ein Meßgerät zur gleichzeitigen, berührungslosen Erfassung von Temperatur und Durchblutungszustand der Haut zur Verfügung. Die Messungen sind komfoltabel durchführbar und gut reproduzierbar, da das Meßregime vom Mikrocontroller objektiviert wird. Eine Eiweiterung des Einsatzgebietes ist auf Meßverfahren denkbar, bei denen Wert auf eine nahezu rückwirkungsfreie Simultanmessung gelegt wird.

Ein geeigneter Kennwert zur Beschreibung des Verlaufs der Wiederdurchblutung ist zu diskutieren.

\section{Literatur}

[1] Hershler, C. et al.: Assessment of an infra-red non-contact sensor for routine skin temperature monitoring: a preliminary study. Journal of Medical Engineering \& Technology 16 (1992), S. $117-122$

[2] Lindberg, L.-G.: Photoplethysmography Part 1: Comparison with laser Doppler flowmetrie. Med. \& Biol. Eng. \& Comput. 29 (1991), S. 40-47.

[3] Lindberg, L.-G.: Photoplethysmography Part 2: influence of light source wavelength. Med. \& Biol. Eng. \& Comput. 29 (1991), S. 48-54.

[4] Steiner, M., Wenzel, A.: Entwicklung der Hardund Software zur Einführung der akralen Wiedererwärmung in die klinische Praxis. Diplomarbeit Humboldt-Universität zu Berlin, 1992. 